\title{
Single shot x-ray diffractometry in SACLA with pulsed magnetic fields up to $16 \mathrm{~T}$
}

\author{
Akihiko Ikeda $\odot,{ }^{*}$ Yasuhiro H. Matsuda $\odot,{ }^{\dagger}$ Xuguang Zhou, and Takeshi Yajima $\odot$ \\ Institute for Solid State Physics, University of Tokyo, Kashiwa, Chiba 277-8581, Japan \\ Yuya Kubota, Kensuke Tono $\odot$, and Makina Yabashi \\ RIKEN SPring-8 Center, Kouto, Soyo, Hyogo 679-5198, Japan \\ and Japan Synchrotron Radiation Research Institute, Kouto, Soyo, Hyogo 679-5198, Japan
}

(Received 28 April 2020; accepted 6 October 2020; published 3 November 2020)

\begin{abstract}
Single shot X-ray diffraction (XRD) experiments have been performed with an X-ray free electron laser under pulsed high magnetic fields up to $16 \mathrm{~T}$ generated with a nondestructive minicoil. The antiferromagnetic insulator phase in a perovskite manganaite, $\operatorname{Pr}_{0.6} \mathrm{Ca}_{0.4} \mathrm{MnO}_{3}$, is collapsed at a magnetic field of $\approx 8 \mathrm{~T}$ with an emergence of the ferromagnetic metallic phase, which is observed via the accompanying lattice changes in a series of the single shot XRD. We discussed the feasibility of the single shot XRD experiment under ultrahigh magnetic fields beyond $100 \mathrm{~T}$, which is generated with a portable destructive pulse magnet.
\end{abstract}

DOI: 10.1103/PhysRevResearch.2.043175

\section{INTRODUCTION}

As a novel light source, an X-ray free electron laser (XFEL) is prominently characterized by the ultrahigh transverse coherence, ultrashort pulses between hundreds to a few femtoseconds and a high-photon flux of $10^{11-12}$ photons/pulse that realizes single shot experiments $[1,2]$. The advent of the XFEL technique has provided us with such new experimental opportunities to explore new areas of science such as coherent diffraction from a single nano-cluster, diffraction before destruction from a living cell, femtosecond time resolution pump-probe experiments, and also experiments with single shot x-ray diffractometry (XRD) under shocked compression environment. We propose an X-ray experiment at an extremely high magnetic field of above $100 \mathrm{~T}$, where we make use of the ultrashort temporal width and the single shot experiment of an XFEL, and a destructive $\mu$ s-pulse magnet for $100 \mathrm{~T}$ generation.

High-field experiments have been successfully combined with synchrotron radiation (SR) based XRD and spectroscopies for condensed matter experiments with DC magnets up to $15 \mathrm{~T}$ [3-7] and nondestructive pulse magnets up to $50 \mathrm{~T}$ [8-16]. The recent advent of single shot techniques with XFELs, further made possible the observations of the weak superlattice reflections of charge density wave order appearing in a cuprate by suppressing the high- $T_{\mathrm{C}}$ superconductivity up to $32 \mathrm{~T}[17,18]$. These high-field studies with SR and XFELs have been confined below $50 \mathrm{~T}$. To generate magnetic fields

\footnotetext{
*ikeda@issp.u-tokyo.ac.jp

†ymatsuda@issp.u-tokyo.ac.jp

Published by the American Physical Society under the terms of the Creative Commons Attribution 4.0 International license. Further distribution of this work must maintain attribution to the author(s) and the published article's title, journal citation, and DOI.
}

over $50 \mathrm{~T}$ is a challenge for conventional portable nondestructive pulse magnet. Nondestructive magnets generating above $50 \mathrm{~T}$ are available only in specific high magnetic field facilities over the world, where pulsed magnetic fields up to $100 \mathrm{~T}$ are available with sufficient safety measures [19], which is for from portable.

For x-ray experiments at well above $50 \mathrm{~T}$, we propose the use of a single turn coil (STC), a destructive pulse magnet, instead of nondestructive pulse magnets, because a STC can be portable and generates magnetic fields over $100 \mathrm{~T}[20,21]$. Generating high fields well beyond $100 \mathrm{~T}$ inevitably requires a destructive pulse magnet where magnetic pressure beyond $4 \mathrm{GPa}$ destroys the coil. For condensed matter experiments, flux compression and single turn coil techniques have been implemented [22-24]. The temporal duration of the generated field by a single turn coil is only a few microseconds, which is about three orders of magnitude smaller than the pulse of a nondestructive magnet. It is still long enough for the single shot experiment with an XFEL pulse. We also refer to an exceptional single shot technique utilizing SR from storage rings [25], which is also usefull with a single shot pulse of $100 \mathrm{~T}$ of a few microseconds.

For a $100 \mathrm{~T}$ experiment at an XFEL site, one needs to construct a portable $100 \mathrm{~T}$ generator. It is profitable to test equipment such as nonmetallic vacuum tubes and cryostats that are conventionally used with the STC experiments. Portable STCs have been implemented before [20,21]. A portable STC to be built should be less than $1000 \mathrm{~kg}$. We are now constructing a portable pulse power with $30 \mathrm{kV}$ charging and $4.5 \mathrm{~kJ}$ energy with a monospark gap. Conventionally, two STCs are in operation at the Institute for Solid State Physics (ISSP), University of Tokyo, with $200 \mathrm{~kJ}$ at $50 \mathrm{kV}$ with a total weight of 10 tons. In ISSP, a specially prepared nonmetallic cryostat and vacuum system has been implemented for destructive pulse experiments at low temperatures. This equipment is proof against the explosions of the STC magnet. It is most preferable to use this equipment in the 
100 T experiments in SPring-8 Angstrom Compact free electron LAser (SACLA). To check this possibility, we need to test if this equipment is compatible with the $\mathrm{X}$-ray experiments in SACLA. Thus, it is fruitful to test the feasibility of this equipment in SACLA with a minicoil mimicking the $100 \mathrm{~T}$ experiment with a portable STC.

In preceding studies for the singe shot XRD at $100 \mathrm{~T}$ in SACLA, we have discovered that the lattices of materials are actually changing under $100 \mathrm{~T}$ fields of a few microseconds pulse generated in destructive pulse magnets [26]. The direct observations of the lattice changes are performed using a high-speed strain sensor utilizing fiber Bragg grating, which is devised for magnetostriction measurements at above $100 \mathrm{~T}$ [27]. A candidate for XRD study at $100 \mathrm{~T}$ is solid oxygen for structural analysis. A high field beyond $120 \mathrm{~T}$ induces a new thermodynamic phase called $\theta$ phase [28]. A cubic lattice geometry is anticipated in the $\theta$ phase in contrast to the monoclinic $\alpha$ phase at low-field phase, which remains elusive until an XRD is performed at $120 \mathrm{~T}$. A magnetostriction measurement found a first-order lattice change accompanying the magnetization jump, indicating a lattice change at the $\alpha-\theta$ transition. Another candidate is a perovskite cobaltite $\mathrm{LaCoO}_{3}$ for investigating the structure and electronic states at $100 \mathrm{~T}$. A peculiar spin crossover is found to be induced by magnetic fields beyond $100 \mathrm{~T}$ [29,30]. There are two high-field phases whose origins are controversially argued to be a Bose-Einstein condensation of excitons, an excitonic insulator, or a crystallization of the spin-state degree of freedom. This is to be determined by observing a superlattice reflection from the spin-state crystallization and also by observing the $d$-electron states by the $\mathrm{x}$-ray emission spectroscopy. Besides them, there are plenty of magnetic phase transitions at ultrahigh magnetic fields where the lattice changes play major roles [31-33].

In this paper, we report an experiment of single shot XRD in SACLA with a nondestructive room-temperaturebore magnet mimicking the single turn coil. The purpose of this work is to see the feasibility of an application of the ready-made explosion-proof equipment for the STC to XRD experiments in SACLA. We successfully detect the fieldinduced phase transition of $\operatorname{Pr}_{0.6} \mathrm{Ca}_{0.4} \mathrm{MnO}_{3}$ with a series of single shot XRD with SACLA up to $16 \mathrm{~T}$. We comment on a design for a portable single turn coil system for SACLA.

\section{EXPERIMENT}

The experiment was performed at a hard x-ray beam line BL2, in SACLA [34,35]. A schematic drawing of the experimental setup is shown in Fig. 1(a). The XFEL was tuned to $16 \mathrm{keV}$ with a mean pulse energy of $\sim 100 \mu \mathrm{J} /$ pulse. A pink beam $\left(\Delta E / E \sim 10^{-3}\right)$ and monochromatic beam $(\Delta E / E \sim$ $\left.10^{-4}\right)$ were used. The single shot diffraction signals were monitored with a multiport charge-coupled device (MPCCD) image sensor [36]. The magnetic fields were generated with a minibank system of $2.4 \mathrm{~kJ}$ at $2000 \mathrm{~V}(1.2 \mathrm{mF})[8,37]$ and a room-temperature coil wound by hand. The waveforms of the pulsed magnetic fields are shown in Fig. 1(b).

The bore of the coil is $1 \mathrm{~cm}$ diameter and $1 \mathrm{~cm}$ long in the axial direction. In the bore, a vacuum tube is suspended with a He-flow and nonmetallic type cryostat located inside as shown in Fig. 1(c). The sample is put inside the cryostat, which is a

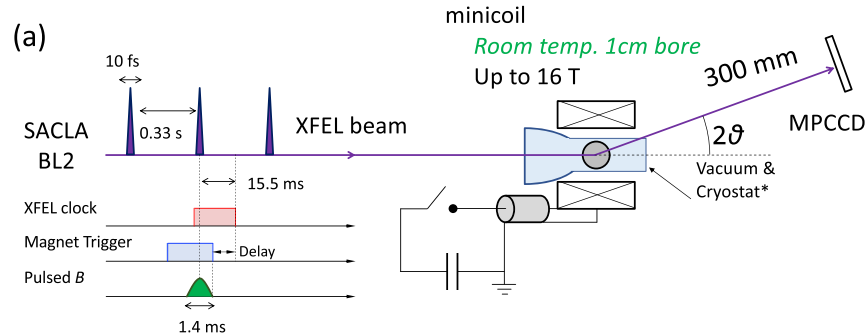

(b)

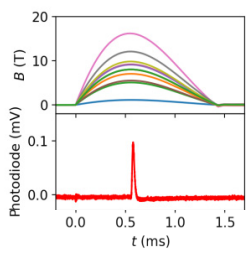

(c)
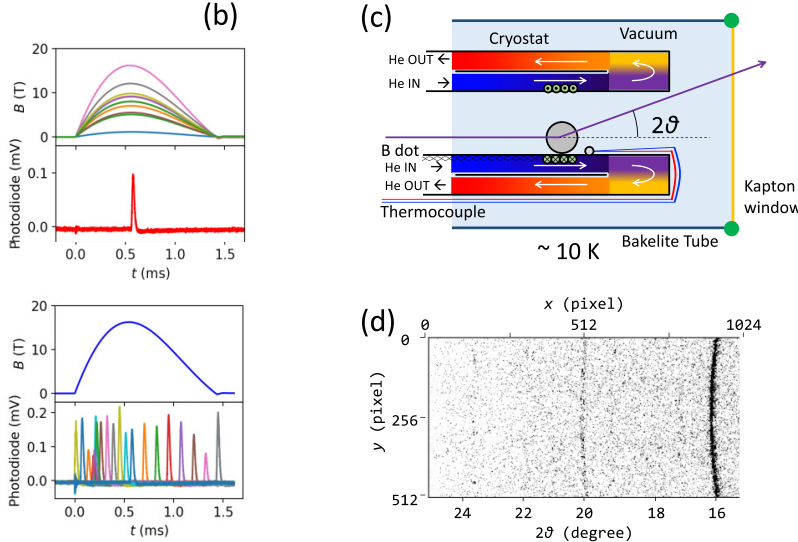

FIG. 1. (a) A schematic drawing of the experimental setup of the single shot XRD in SACLA with a pulse magnet. The temporal diagram of the measurement is also shown. (b) The time evolution of the magnetic field and the XFEL pulse intensity. Magnetic fields can be varied in two ways in a single shot XRD. The generated magnetic field is varied with a fixed XFEL timing on top of the pulsed magnetic field. In another way, the XFEL timing is varied with a fixed waveform of the pulsed magnetic field. (c) A schematic drawing of a part of the nonmetallic cryostat used in ISSP for STC experiments, which is used this time for XRD study for verification of the compatibility. (d) An image of a single shot XRD recorded by the MPCCD image sensor with a monochromatic beam of the XFEL.

position where an XFEL pulse hits and the diffracted signal escapes through Kapton windows at the back and top of the vacuum chamber. The $2 \theta$ range of $15^{\circ}-25^{\circ}$ is captured with a $25.6 \times 51.2 \mathrm{~mm}$ window of the MPCCD with a distance of $300 \mathrm{~mm}$ from the sample to the detector. A single shot XRD image is shown in Fig. 1(d).

$\mathrm{Pr}_{0.6} \mathrm{Ca}_{0.4} \mathrm{MnO}_{3}$ was powdered from a single crystal and dispersed in a glue whose effective thickness was $\sim 10 \mu \mathrm{m}$. The powder was only roughly powdered on purpose so that a spotty Debye ring is observed in XRD, where it is expected that we observe a speckle pattern around the diffraction spot reflecting the microstrain of a particle.

\section{RESULT AND DISCUSSION}

Figure 2 shows a series of single shot XRD at various magnetic fields up to $16.3 \mathrm{~T}$ at $2 \theta \simeq 16.3^{\circ}$, where 020,200 , and 112 reflections are relevant. The spotty diffraction ring is produced from the roughly powdered sample. One diffraction spot is coming from a single domain of a microparticle of a sample. The magnetic field effect is visible in Fig. 2. The trends of the images are similar to each other from 0 to $6.6 \mathrm{~T}$. The images from 9.7 to $16.3 \mathrm{~T}$ have a common feature being 


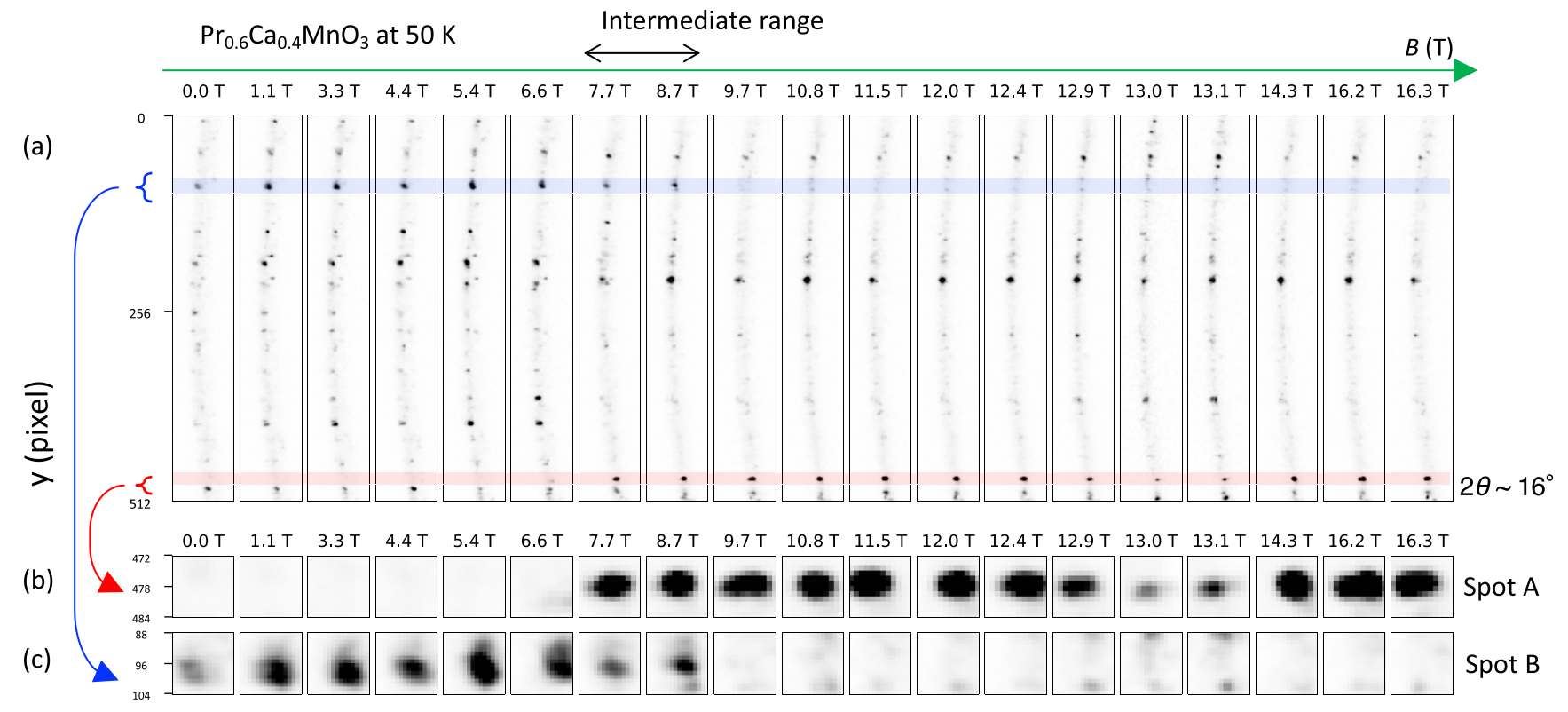

FIG. 2. (a) Image of a series of single shot $\mathrm{XRD}$ at $2 \theta \simeq 16^{\circ}$ from a rough powdered sample of $\operatorname{Pr}_{0.6} \mathrm{Ca}_{0.4} \mathrm{MnO}_{3}$ at $50 \mathrm{~K}$ with various magnetic fields up to $16.3 \mathrm{~T}$ using a pink beam of an XFEL in SACLA at $h v=16 \mathrm{keV}$. (b) Magnification of spot A and (c) spot B.

distinct from the low-field data. The images at 7.7 and $8.7 \mathrm{~T}$ seem to be linear combinations of both features from low-field and high-field data, indicating a transient state. Two magnifications are shown in Figs. 2(b) and 2(c). In Fig. 2(b), it is clear that spot A appears above 7.7 T. In Fig. 2(c), on the contrary, spot B disappears above 9.7 T. The x-ray intensities of spot A and spot $\mathrm{B}$ are shown as a function of $B$ as shown in Fig. 3. The appearance of spot $\mathrm{A}$ means that the $\theta-2 \theta$ configuration is satisfied after the field-induced lattice parameter change of the particle for spot A. In contrast, for spot B, which vanishes after the field-induced lattice parameter change of the particle for spot $\mathrm{B}$, the $\theta-2 \theta$ configuration is no longer satisfied at high fields. Here we have used the pink beam, where the energy dispersion of $10^{-3}$ results in a too large uncertainty in $2 \theta$ for the assignment of the diffraction peaks. Note that the data points at 13.0 and $13.1 \mathrm{~T}$ behave strangely both in Figs. 2(a)-2(c) and 3. The origin of the behavior is unclear but possibly intrinsic to the sample, considering that the regular calibration procedures have been properly carried out for the

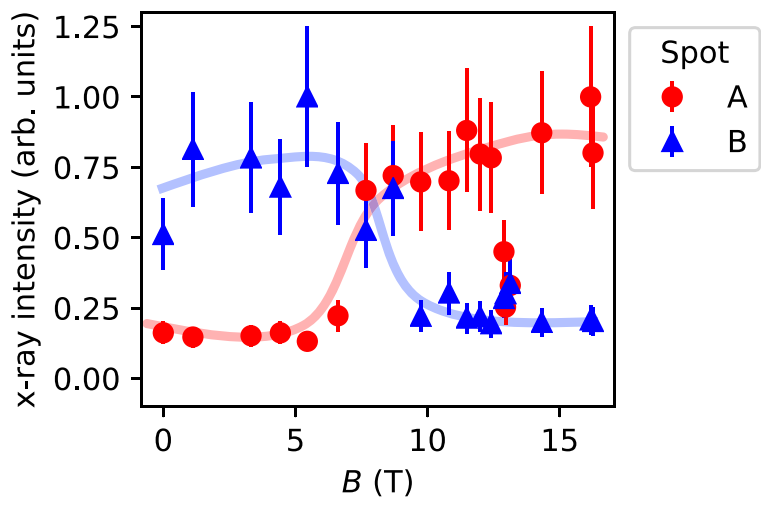

FIG. 3. X-ray intensity of spots A and B of Figs. 2(b) and 2(c) as a function of external magnetic fields. instabilities of the intensity and the wavelength of the XFEL pulses.

For high-resolution measurements in the diffraction angle, we have performed a series of single shot XRD at pulsed high magnetic fields with a monochromated XFEL beam. The result is shown in Fig. 4(a). The diffraction peaks at $16.36^{\circ}$
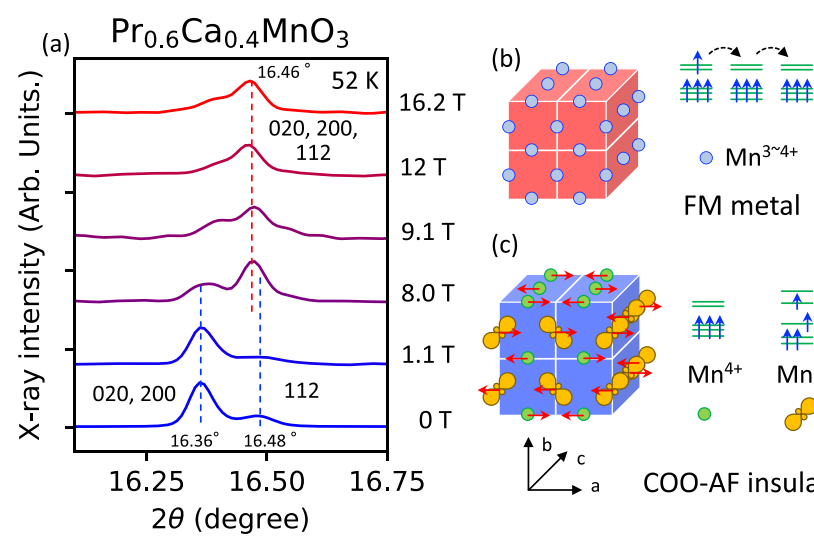

(c)

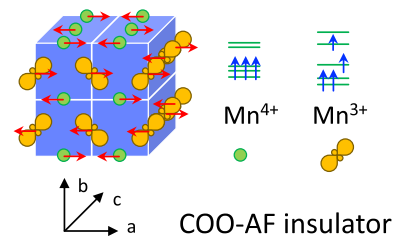

FIG. 4. (a) A series of single shot XRD at $2 \theta \simeq 16^{\circ}$ with a monochromated XFEL beam at $h v=16 \mathrm{keV}$ from a powdered sample of $\operatorname{Pr}_{0.6} \mathrm{Ca}_{0.4} \mathrm{MnO}_{3}$ at $52 \mathrm{~K}$ at various magnetic fields up to $16.3 \mathrm{~T}$. (b) A schematic drawing of the FM metallic phase of $\operatorname{Pr}_{0.6} \mathrm{Ca}_{0.4} \mathrm{MnO}_{3}$ at above $8 \mathrm{~T}$ at $52 \mathrm{~K}$. It is depicted that the electron in the $e_{g}$ orbitals are delocalized and that the double exchange scheme favors the ferromagnetic spin configurations of the localized $t_{2 g}$ orbitals, due to the Hund's coupling between the delocalized $e_{g}$ electrons and the localized $t_{2 g}$ electrons. (c) A schematic drawing of the COOI phase of $\mathrm{Pr}_{0.6} \mathrm{Ca}_{0.4} \mathrm{MnO}_{3}$ at below $8 \mathrm{~T}$ at $52 \mathrm{~K}$, depicting the charge, orbital, and spin configurations, which is reproduced from Ref. [38]. The localized electron occupies the $3 z^{2}-r^{2}$ orbital at the $\mathrm{Mn}^{3+}$ site, which is stable via the Jahn-Teller effect. The antiferromagnetic spin configuration of so-called CE type is depicted. 
and $16.48^{\circ}$ are identified as 020,200 , and 112 reflections in a Pbmn setting, respectively. It is clear that the peak at $16.36^{\circ}$ decreases at above $8 \mathrm{~T}$, while the new peak at $16.46^{\circ}$ increases. This behavior is in good agreement with the previous result with SR [8]. In $\mathrm{Pr}_{0.6} \mathrm{Ca}_{0.4} \mathrm{MnO}_{3}$, with decreasing temperature, the phase transition occurs from a ferromagnetic metallic (FM) phase to the charge and orbital ordered insulator (COOI) phase [39]. When the COOI phase appears, the $a$ and $b$ axes elongate and the $c$ axis shrinks, stabilizing the occupied $3 z^{2}-r^{2}$ orbital state $[38,40]$. The COOI phase is accompanied by antiferromagnetism. Thereby, it can be collapsed with an external magnetic field. With an emergence of the magnetic field-induced FM phase, the $a$ and $b$ axes shrink, and the $c$ axis elongates. This is schematically depicted in Figs. 4(b) and 4(c). According to this picture, the 020 and 200 diffractions are supposed to shift to higher $2 \theta$ overlapping the peak of 112, and the 112 diffraction stays. As a result, the diffraction peak at $16.38^{\circ}$ decreases and the diffraction at $16.46^{\circ}$ increases. The observation in Fig. 4 is in good agreement with the above expectation, indicating that the field-induced COOI with a single shot XRD in SACLA is successfully observed.

So far it is shown that the single shot XRD is successfully conducted with a 1.4-ms pulsed magnetic field and the nonmetallic cryostat and the vacuum tube that are compatible with the STC experiments. Also, the lattice change of $\operatorname{Pr}_{0.6} \mathrm{Ca}_{0.4} \mathrm{MnO}_{3}$ accompanying the field-induced phase transition is observed in a series of single shot XRD experiments. The observed changes of XRD are consistent with the shrinkage of the $a$ and $b$ axes and an elongation of the $c$ axis at high fields.

Here, we propose and discuss the feasibility of a single shot powder XRD measurement up to $100 \mathrm{~T}$ for a structural analysis. In the present study, a rough powdered sample was employed with the motivation to observe speckle patterns from a micrograin under stress in the two phase coexistence state during the phase transition. However, such speckle patterns are not observed around the diffraction spots in Fig. 2. The $100 \mathrm{~T}$ XRD experiment will focus on the structural analysis by means of the smooth powder diffraction using a well powdered sample. In the present study, a limited range of $2 \theta<25^{\circ}$ is covered, where a small number of low indexed diffraction peaks are obtained, being limited by the opening angles of the fiber-reinforced plastic (FRP) based cryostat and the minicoil. The dimensions of the present minicoil are $d=10 \mathrm{~mm}$ in diameter and $h=10 \mathrm{~mm}$ in axial length. For structural analysis, it is favorable to cover a larger angle up to $2 \theta \sim 60^{\circ}$. A larger coverage of $2 \theta$ becomes possible with the use of an all-Kapton cryostat and a vacuum tube with a Kapton window as shown in Fig. 5. In the $100 \mathrm{~T}$ XRD experiment, a double turn coil is to be used which has a larger aspect ratio of $h / d=\sim 0.5$. This allows us to cover $2 \theta$ up to $60^{\circ}$ as schematically inspected in Fig. 5.

Presently, the monochromated beam shows an appreciable resolution of XRD for the structural analysis, while the pink beam does not. The use of the narrow and brighter beam of the seeded beam [41] is even more practical in the $100 \mathrm{~T}$ $\mathrm{XRD}$ experiment. In addition, the use of a photon energy of $10 \mathrm{keV}$ increases the photon number by a factor of $\sim 6$ instead of a photon energy of $16 \mathrm{keV}$ used in the present study. Presently, $h v=16 \mathrm{keV}$ is used due to the limitation of the

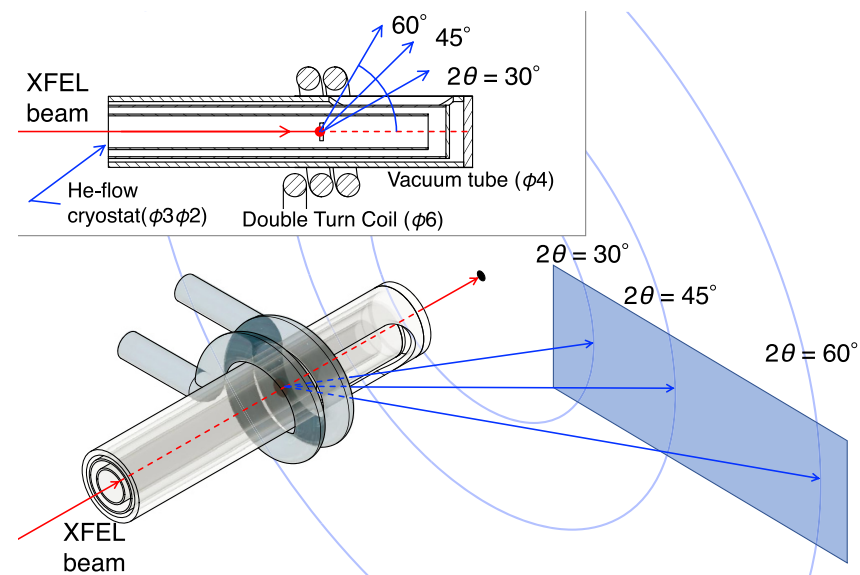

FIG. 5. A schematic view of a proposed powder diffraction experiment in SACLA with a double turn coil, generating up to $80 \mathrm{~T}$. The diffraction angles $2 \theta=30^{\circ}, 45^{\circ}, 60^{\circ}$ are shown. The Debye ring is projected on a plane $1.4 \mathrm{~cm}$ away from the diffraction center. The double turn coil, the vacuum tube, and the He-flow cryostat are made of copper, Kapton tube, and FRP with a Kapton window for the diffracted $\mathrm{x}$-ray. The inset is a cross-sectional view of the double turn coil, the vacuum tube, and the cryostat, showing the opening angle for the diffracted $\mathrm{x}$ ray. The shaded area colored in light blue represents the projection of the coverage by an IP with the size of $20 \times 40 \mathrm{~cm}$ located $30 \mathrm{~cm}$ away from the diffraction center.

small $2 \theta$ range. With the larger $2 \theta$ as proposed above, the use of $h v=10 \mathrm{keV}$ is allowed. A magnetic field of $80 \mathrm{~T}$ is estimated to be obtained with the double turn coil with a diameter of $6 \mathrm{~mm}$ and the portable bank system of ours currently under construction, which is rated at $30 \mathrm{kV}$ with an energy of $4.5 \mathrm{~kJ}$, generating $200 \mathrm{kA}$. Instead of the MPCCD detector, we plan to use a conventional $\mathrm{x}$-ray imaging plate (IP). This is because an IP allows a much more robust detection against a fragmentation of the coil, which is not feasible with the fragile MPCCD detectors.

The single-crystalline experiment aiming for a weak structurelike superlattice reflection is at this moment not feasible due to the limited volume $\left(<5 \times 5 \times 5 \mathrm{~mm}^{3}\right)$ of the magnetic field generated in STC, where a sample rotation mechanism should be somehow installed. Evaluation of this method will be a future task.

\section{SUMMARY}

We performed single shot XRD experiments in SACLA with a pulsed magnetic field up to $16 \mathrm{~T}$. We have successfully observed a field-induced lattice change in $\operatorname{Pr}_{0.6} \mathrm{Ca}_{0.4} \mathrm{MnO}_{3}$, which is originated in a collapse of charge and orbital ordered insulator phase and the appearance of a ferromagnetic metallic phase. Based on this result, a methodology of a single shot XRD experiment up to $100 \mathrm{~T}$ range in SACLA using a portable $100 \mathrm{~T}$ generator is discussed.

\section{ACKNOWLEDGMENTS}

This work was supported by JSPS KAKENHI Grant-inAid for Young Scientists Grant No. 18K13493, JSPS KAKENHI Challenging Research (Pioneering) No. 20K20521, and the Basic Science Program Grant No. 18-001 of TEPCO 
Memorial Foundation. We thank T.-h. Arima for providing us with a high-quality single-crystalline sample. The present experiment was performed with the approval of the
Japan Synchrotron Radiation Research Institute (Proposal No. 2019A8017). The authors would like to acknowledge the supporting members of the SACLA facility.
[1] C. Bostedt, S. Boutet, D. M. Fritz, Z. Huang, H. J. Lee, H. T. Lemke, A. Robert, W. F. Schlotter, J. J. Turner, and G. J. Williams, Rev. Mod. Phys. 88, 015007 (2016).

[2] E. A. Seddon, J. A. Clarke, D. J. Dunning, C. Masciovecchio, C. J. Milne, F. Parmigiani, D. Rugg, J. C. H. Spence, N. R. Thompson, K. Ueda, S. M. Vinko, J. S. Wark, and W. Wurth, Rep. Prog. Phys. 80, 115901 (2017).

[3] V. Kiryukhin and B. Keimer, Phys. Rev. B 52, R704(R) (1995).

[4] V. Kiryukhin, B. Keimer, and D. E. Moncton, Phys. Rev. Lett. 74, 1669 (1995).

[5] V. Kiryukhin, B. Keimer, J. P. Hill, and A. Vigliante, Phys. Rev. Lett. 76, 4608 (1996).

[6] Y. Narumi, K. Katsumata, Y. Tabata, S. Kimura, Y. Tanaka, T. Nakamura, S. Shimomura, M. Matsuda, I. Harada, Y. Nishiyama et al., J. Phys. Soc. Jpn. 73, 2650 (2004).

[7] Y. Narumi, K. Katsumata, U. Staub, K. Kindo, M. Kawauchi, C. Broennimann, H. Toyokawa, Y. Tanaka, A. Kikkawa, T. Yamamoto, M. Hagiwara, T. Ishikawa, and H. Kitamura, J. Phys. Soc. Jpn. 75, 075001 (2006).

[8] Y. H. Matsuda, Y. Ueda, H. Nojiri, T. Takahashi, T. Inami, K. Ohwada, Y. Murakami, and T. Arima, Phys. B (Amsterdam, Neth.) 346, 519 (2004).

[9] Y. Narumi, K. Kindo, K. Katsumata, M. Kawauchi, C. Broennimann, U. Staub, H. Toyokawa, Y. Tanaka, A. Kikkawa, T. Yamamoto, M. Hagiwara, T. Ishikawa, and H. Kitamura, J. Synchrotron Radiat. 13, 271 (2006).

[10] Y. H. Matsuda, Z. W. Ouyang, H. Nojiri, T. Inami, K. Ohwada, M. Suzuki, N. Kawamura, A. Mitsuda, and H. Wada, Phys. Rev. Lett. 103, 046402 (2009).

[11] M. Sikora, O. Mathon, P. van der Linden, J. M. Michalik, J. M. de Teresa, C. Kapusta, and S. Pascarelli, Phys. Rev. B 79, 220402(R) (2009).

[12] J. P. C. Ruff, Z. Islam, J. P. Clancy, K. A. Ross, H. Nojiri, Y. H. Matsuda, H. A. Dabkowska, A. D. Dabkowski, and B. D. Gaulin, Phys. Rev. Lett. 105, 077203 (2010).

[13] Y. H. Matsuda, J. L. Her, S. Michimura, T. Inami, M. Suzuki, N. Kawamura, M. Mizumaki, K. Kindo, J. Yamauara, and Z. Hiroi, Phys. Rev. B 84, 174431 (2011).

[14] Z. Islam, D. Capatina, J. P. Ruff, R. K. Das, E. Trakhtenberg, H. Nojiri, Y. Narumi, U. Welp, and P. C. Canfield, Rev. Sci. Instrum. 83, 035101 (2012).

[15] J. Billette, F. Duc, P. Frings, M. Nardone, A. Zitouni, C. Detlefs, T. Roth, W. Crichton, J. E. Lorenzo, and G. L. Rikken, Rev. Sci. Instrum. 83, 043904 (2012).

[16] Y. H. Matsuda and T. Inami, J. Phys. Soc. Jpn. 82, 021009 (2013).

[17] S. Gerber, H. Jang, H. Nojiri, S. Matsuzawa, H. Yasumura, D. A. Bonn, R. Liang, W. N. Hardy, Z. Islam, A. Mehta et al., Science 350, 949 (2015).

[18] H. Jang, W. S. Lee, H. Nojiri, S. Matsuzawa, H. Yasumura, L. Nie, A. V. Maharaj, S. Gerber, Y. J. Liu, A. Mehta et al., Proc. Natl. Acad. Sci. USA 113, 14645 (2016).
[19] R. Battesti, J. Beard, S. Böser, N. Bruyant, D. Budker, S. A. Crooker, E. J. Daw, V. V. Flambaum, T. Inada, I. G. Irastorza et al., Phys. Rep. 765-766, 1 (2018).

[20] F. Herlach, R. McBroom, T. Erber, J. Murray, and R. Gearhart, IEEE Trans. Nucl. Sci. 18, 809 (1971).

[21] O. Portugall, N. Puhlmann, H. U. Müller, M. Barczewski, I. Stolpe, M. Thiede, H. Scholz, M. v. Ortenberg, and F. Herlach, J. Phys. D: Appl. Phys 30, 1697 (1997).

[22] F. Herlach, Rep. Prog. Phys. 62, 859 (1999).

[23] N. Miura, T. Osada, and S. Takeyama, J. Low Temp. Phys. 133, 139 (2003).

[24] D. Nakamura, A. Ikeda, H. Sawabe, Y. H. Matsuda, and S. Takeyama, Rev. Sci. Instrum. 89, 095106 (2018).

[25] Note an exceptional technique where single shot XRD is utilized with a bright and broadband SR from a storage ring for shock physics experiments [42-47].

[26] A. Ikeda, Y. H. Matsuda, D. Nakamura, S. Takeyama, H. Tsuda, K. Nomura, A. Shimizu, A. Matsuo, T. Nomura, T. C. Kobayashi, T. Yajima, H. Ishikawa, Z. Hiroi, M. Isobe, T. Yamauchi, and K. Sato, in Proceedings of the 2018 16th International Conference on Megagauss Magnetic Field Generation and Related Topics (MEGAGAUSS), Kashiwa, Japan (IEEE, Piscataway, NJ, 2019), pp. 1-9.

[27] A. Ikeda, T. Nomura, Y. H. Matsuda, S. Tani, Y. Kobayashi, H. Watanabe, and K. Sato, Rev. Sci. Instrum. 88, 083906 (2017).

[28] T. Nomura, Y. H. Matsuda, S. Takeyama, A. Matsuo, K. Kindo, J. L. Her, and T. C. Kobayashi, Phys. Rev. Lett. 112, 247201 (2014).

[29] A. Ikeda, T. Nomura, Y. H. Matsuda, A. Matsuo, K. Kindo, and K. Sato, Phys. Rev. B 93, 220401(R) (2016).

[30] A. Ikeda, Y. H. Matsuda, and K. Sato, Phys. Rev. Lett. 125 , 177202 (2020).

[31] A. Miyata, H. Ueda, Y. Ueda, H. Sawabe, and S. Takeyama, Phys. Rev. Lett. 107, 207203 (2011).

[32] T. T. Terashima, A. Ikeda, Y. H. Matsuda, A. Kondo, K. Kindo, and F. Iga, J. Phys. Soc. Jpn. 86, 054710 (2017).

[33] Y. H. Matsuda, D. Nakamura, A. Ikeda, S. Takeyama, Y. Suga, H. Nakahara, and Y. Muraoka, Nat. Commun. 11, 3591 (2020).

[34] T. Ishikawa, H. Aoyagi, T. Asaka, Y. Asano, N. Azumi, T. Bizen, H. Ego, K. Fukami, T. Fukui, Y. Furukawa et al., Nat. Photon. 6, 540 (2012).

[35] M. Yabashi, H. Tanaka, and T. Ishikawa, J. Synchrotron Radiat. 22, 477 (2015).

[36] T. Kameshima, S. Ono, T. Kudo, K. Ozaki, Y. Kirihara, K. Kobayashi, Y. Inubushi, M. Yabashi, T. Horigome, A. Holland, K. Holland, D. Burt, H. Murao, and T. Hatsui, Rev. Sci. Instrum. 85, 033110 (2014).

[37] Y. H. Matsuda, T. Inami, K. Ohwada, Y. Murata, H. Nojiri, Y. Murakami, H. Ohta, W. Zhang, and K. Yoshimura, J. Phys. Soc. Jpn. 76, 034702 (2007).

[38] Y. Tokura and Y. Tomioka, J. Magn. Magn. Mater. 200, 1 (1999).

[39] Y. Tomioka, A. Asamitsu, H. Kuwahara, Y. Moritomo, and Y. Tokura, Phys. Rev. B 53, R1689(R) (1996). 
[40] H. Kuwahara, Y. Tomioka, A. Asamitsu, Y. Moritomo, and Y. Tokura, Science 270, 961 (1995).

[41] I. Inoue, T. Osaka, T. Hara, T. Tanaka, T. Inagaki, T. Fukui, S. Goto, Y. Inubushi, H. Kimura, R. Kinjo et al., Nat. Photon. 13, 319 (2019).

[42] S. J. Turneaure, N. Sinclair, and Y. M. Gupta, Phys. Rev. Lett. 117, 045502 (2016).

[43] S. J. Turneaure, S. M. Sharma, T. J. Volz, J. M. Winey, and Y. M. Gupta, Sci. Adv. 3, eaao3561 (2017).
[44] S. J. Tracy, S. J. Turneaure, and T. S. Duffy, Phys. Rev. Lett. 120, 135702 (2018).

[45] S. J. Turneaure, P. Renganathan, J. M. Winey, and Y. M. Gupta, Phys. Rev. Lett. 120, 265503 (2018).

[46] S. J. Turneaure, S. M. Sharma, and Y. M. Gupta, Phys. Rev. Lett. 121, 135701 (2018).

[47] K. Ichiyanagi, S. Takagi, N. Kawai, R. Fukaya, S. Nozawa, K. G. Nakamura, K. D. Liss, M. Kimura, and S. I. Adachi, Sci. Rep. 9, 7604 (2019). 